\title{
Digital Beamforming for HRWS-SAR Imaging System Design, Performance and Optimization Strategies
}

\author{
Nicolas Gebert, Gerhard Krieger, Alberto Moreira \\ Microwaves and Radar Institute, DLR (German Aerospace Center), Oberpfaffenhofen, Germany \\ Email: nico.gebert@dlr.de
}

\begin{abstract}
Multi-aperture synthetic aperture radar (SAR) systems in combination with an appropriate coherent processing of the individual aperture signals enable high resolution wide swath (HRWS) SAR imaging [1]-[8]. An innovative reconstruction algorithm for such a digital beamforming on receive was presented in [9]-[12] that allows for HRWS even in case of a non-uniformly sampled data array in azimuth. This paper will compare this algorithm to different azimuth processing strategies regarding their performance in dependency of the overall sampling. Further, optimization strategies are discussed to maximize the system's performance by pattern tapering on transmit and "Pre-Beamshaping on Receive" networks that allow for pattern tapering on receive and adaptively adjust the virtual sample positions.
\end{abstract}

\section{Introduction}

\subsection{Multi-Aperture Sampling}

Several innovative techniques using multiple receive apertures (' $R x$ ') have been suggested to overcome the inherent limitations of conventional SAR to perform HRWS imaging [1]-[8]. For optimum performance the relation between sensor velocity $v$ and the along-track offsets $\Delta x$ of the $N$ sub-apertures has to result in equally spaced effective phase centers thus leading to a uniform sampling of the received signal (cf. Fig. 1, left). This requires the following relation:

$$
P R F_{o p t}=\frac{2 \cdot v}{N \cdot \Delta x}
$$

If a non-optimum $P R F$ is chosen, the gathered samples are spaced non-uniformly. This requires a further processing step after down-conversion and quantization of the multi-aperture azimuth signal before conventional monostatic focusing algorithms can be applied. For this, the individual aperture signals are regarded as independent Rx channels (cf. Fig. 1, right). The purpose of the azimuth processing is to combine the $N$ channels, each of bandwidth $N \cdot P R F$ but sub-sampled with $P R F$, to obtain a signal effectively sampled with $N \cdot P R F=P R F_{\text {eff }}$. Thus the Nyquist criterion is fulfilled in average after the processing, which yields - ideally - an output signal that is free of aliasing.

\section{Azimuth Processing}

\subsection{Algorithms}

In the following, three more methods to process the azimuth signal of a multi-aperture signal are presented and compared to the „reconstruction algorithm“.

1) Displaced Phase Center Antenna (DPCA): This technique proposes to recover the azimuth signal by interleaving the samples of the different Rx channels without any further processing [1]. Consequently, the stringent timing requirement of Eq. (1) has to be fulfilled to obtain a signal that is equivalent to a monostatic signal of $N$ times the $P R F$. In any other case the sample positions deviate from the ideal positions, but they are treated as if the signal was sampled uniformly.

2) Phase Correction: This method takes the properties of the SAR signal into account. It is based on an analysis of the multi-aperture signal's phase compared to the phase of a monostatic and uniformly sampled signal. This yields a phase difference depending on Doppler frequency. By applying an appropriate phase correction to the multi-aperture data, its phase is adjusted in a way such that the resulting phase corresponds to the monostatic and uniformly sampled signal. [3]

3) The Reconstruction Algorithm is based on solving a system of linear equations to unambiguously recover the formerly aliased azimuth spectrum. A detailed derivation and investigation can be found in [9]-[12]. As already indicated in [9], this method comprises the approach in 4) and leads to nearly identical results in a single platform system.

4) Null-Steering: This space-time approach is based on adaptively adjusting the weighting coefficients of the azimuth chan-
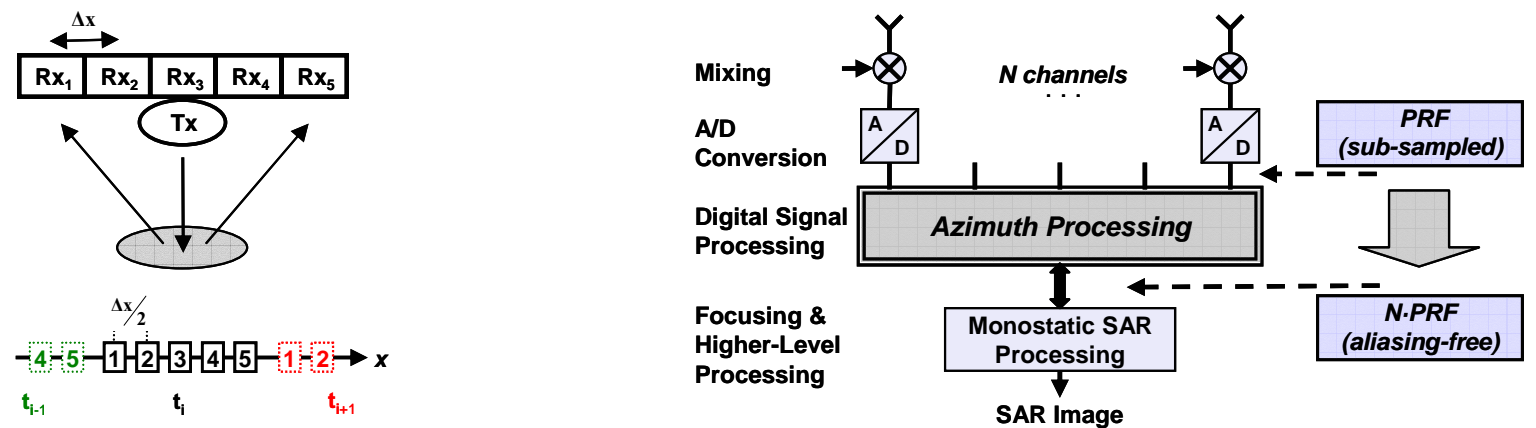

Fig. 1: Left: Multi-Aperture System consisting of $5 \mathrm{Rx}$ apertures and a separate Tx antenna and corresponding virtual sample positions for subsequent pulses $t_{i-1}, t_{i}, t_{i+1}$. Right: Block diagram of the processing. After down-converting and digitizing every aperture's signal, the azimuth processing combines $N$ aliased channels to one output signal with $N$ times the original sampling ratio. 
nels to steer the nulls in the resulting joint antenna pattern to the angles corresponding to the ambiguous Doppler frequencies. This corresponds to a spatial filtering of the data to suppress ambiguous frequencies in the azimuth signal. [13]

\subsection{Reference System}

Fig. 1, left and Table I resume the main parameters of the simulated system. The Rx antenna pattern was approximated by a $\sin (x) / x$ while a Bessel-function was used for the Tx antenna.

\begin{tabular}{|l|c|c|}
\hline \multicolumn{1}{|c|}{ Parameter } & Symbol & Value \\
\hline Carrier wavelength & $\lambda$ & $3.1 \mathrm{~cm}$ \\
\hline Mean slant range & $r_{0}$ & $680 \mathrm{~km}$ \\
\hline Swath-width & $L_{s w}$ & $80 \mathrm{~km}$ \\
\hline Rx Sub-apertures in azimuth & $N$ & 5 \\
\hline Rx Sub-aperture length in azimuth & $d_{a, r x}$ & $3.2 \mathrm{~m}$ \\
\hline Tx Dish antenna in azimuth & $d_{a, t x}$ & $3.5 \mathrm{~m}$ \\
\hline Sensor velocity & $v$ & $7600 \mathrm{~m} / \mathrm{s}$ \\
\hline Processed azimuth bandwidth & $B_{D o p}$ & $5.064 \mathrm{~Hz}$ \\
\hline
\end{tabular}

TABLE I. Main parameters of the investigated HRWS SAR system.

\subsection{Performance}

The performance analysis is done by simulating the point target response resulting from the different processing methods. It is performed for a varying $P R F$ of $1 \mathrm{kHz}$ to $2 \mathrm{kHz}$ what corresponds to varying sampling scenarios of the system.

\subsubsection{Resolution (3-dB width of power)}

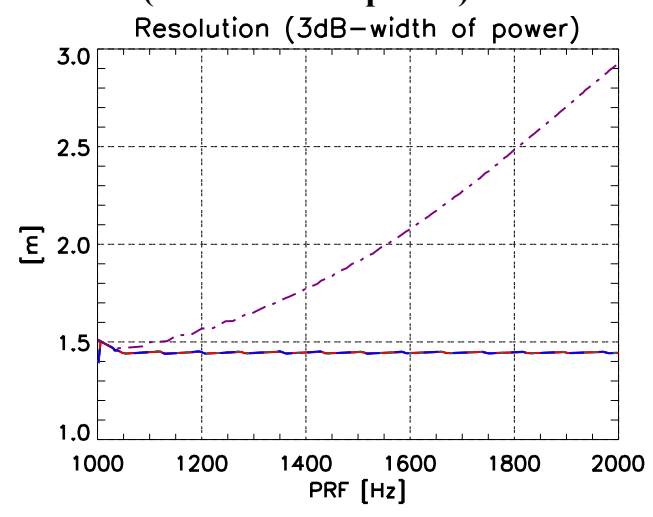

Fig. 2. Resolution (3-dB width of the squared amplitude of the impulse response) vs. PRF. Phase-Correction (Dotted green), Reconstruction (red) and Null-Steering (dashed blue) provide a constant resolution of $1.45 \mathrm{~m}$ while it degrades for DPCA (dotted-dashed violet) for increasing $P R F$.

\subsubsection{Azimuth Ambiguity Suppression}

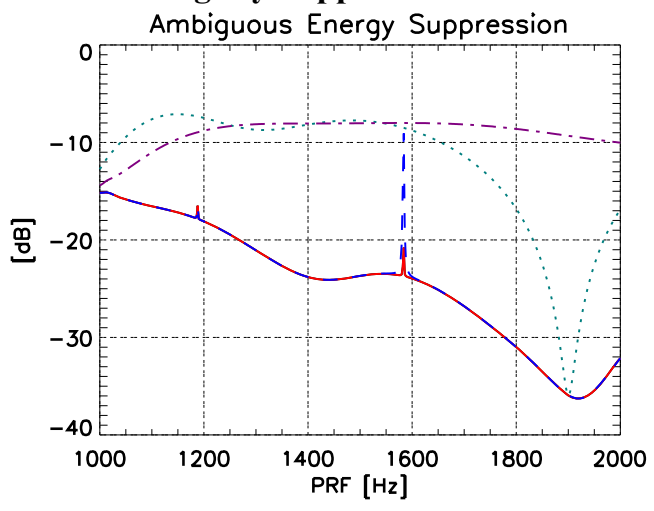

Fig. 3. Ambiguity suppression vs. PRF. Phase Correction (Dotted green) and DPCA (dotted-dashed violet) show significantly worse suppression than Reconstruction (red) and Null-Steering (dashed blue) that are nearly identical.
The ambiguous energy suppression represents the logarithmic ratio of the integrated energy of the first 10 ambiguities to the 'real' target's energy of the impulse response. For $1188 \mathrm{~Hz}$ and $1583 \mathrm{~Hz}$ samples of different sub-apertures coincide spatially causing the processing to fail what results in the peaks. A simple solution to this problem could be to combine the RF signals of the respective coinciding channels yielding a more suitable sampling scheme for the processing. (See also 3.2.)

\subsubsection{SNR / Scaling of Noise Power}

As presented in [11], the azimuth processing with the reconstruction algorithm may cause a rise of the noise floor with increasing non-uniformity of the sampling showing singular values for the critical PRFs mentioned in 2.3.2 (cf. Fig. 4). The same problem occurs for the null-steering approach while in the case of the Phase Correction method the noise floor remains constant. Regarding the DPCA approach, the noise power is not increased but a clear loss of peak power of the focused target is observed for increasing non-uniform sampling.

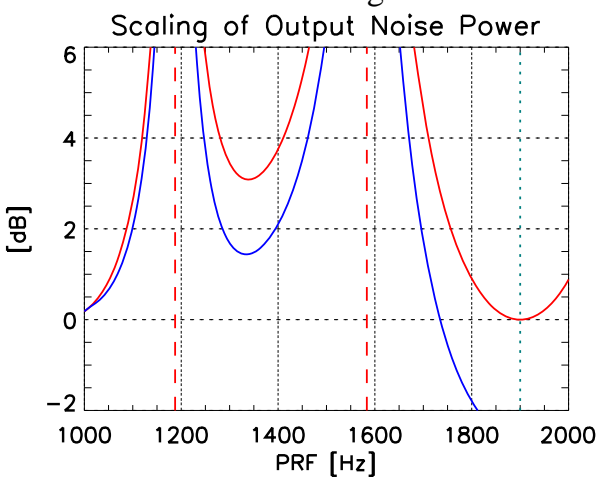

Fig. 4. Scaling of Noise Power vs. PRF before focusing (red) and afterwards (blue) considering the gain due to over-sampling.

\subsubsection{Summary Azimuth Processing}

We have seen that the more sophisticated approaches like nullsteering and reconstruction algorithm provide efficient ambiguity suppression and constant resolution over a varying $P R F$, while the other methods fail. But as indicated in 2.3.1, a possible rise of noise power depending on the overall sampling has to be considered.

\subsection{Adaptation of Sensor Dimensions}

In a next step, the system presented in 2.2 is modified to obtain improved ambiguity suppression and mitigate the inconveniences arising from the scaling of the noise power. The modification comprises a $\mathrm{Rx}$ aperture that is shortened to 2.8 from $3.2 \mathrm{~m}$ and the enlargement of the Tx-dish from 3.5 to $5 \mathrm{~m}$. The new overall $\mathrm{Rx}$ antenna length of 14 m yields an optimum $P R F$ of $1085 \mathrm{~Hz}$ that is adapted to the timing ${ }^{1}$ what limits the maximum scaling of the output noise in the considered interval ${ }^{1}$ to 2 $\mathrm{dB}$ before and $1 \mathrm{~dB}$ after focusing. In addition to that, the increased dish size results in clearly improved ambiguity suppression over a wide $P R F$-range (cf. Fig. 5) at the expense of a

\footnotetext{
${ }^{1}$ An analysis of the timing diagram of the system (orbit height $530 \mathrm{~km}$ ) yields a necessary $P R F$ range from 1.1 to $1.25 \mathrm{kHz}$ to cover completely the incident angle range from $20-50^{\circ}$ with a swath-width of $80 \mathrm{~km}$.
} 
slight increase of resolution from 1.45 to $1.55 \mathrm{~m}$ in the modified system.

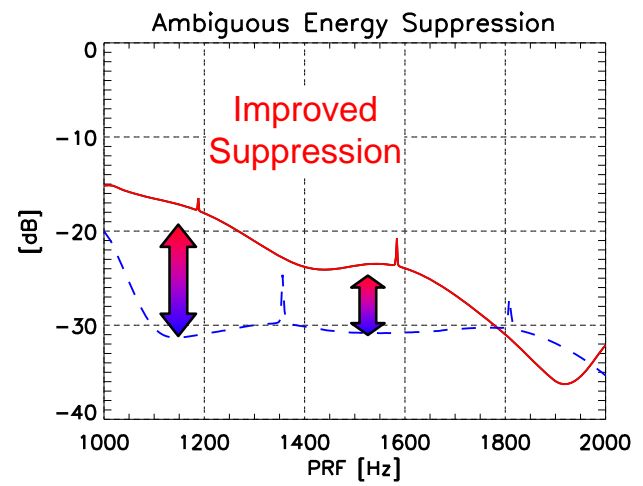

Fig. 5. Ambiguity suppression vs. PRF. Improved suppression of the modified system (dashed blue) compared to the original system (solid red) over a wide range of $P R F$.

\section{Optimized System Concepts}

\subsection{Pattern Tapering on Transmit}

The following chapter presents an approach to improve the system performance by adapting the Tx branch. As derived in [14], all spectral signal energy outside the band $[-N \cdot P R F / 2$, $N \cdot P R F / 2]$ gives rise to ambiguities. This can be avoided by confining the Doppler bandwidth of the signal to $N \cdot P R F$ by an appropriate antenna pattern. As enlarging the $\mathrm{Tx}$ antenna achieves this only at the expense of resolution (cf. 2.4), an adapted tapering can provide improved ambiguity suppression without degrading resolution. To demonstrate the potential of tapering, we consider the system of 2.4 and investigate different combinations of Tx antennas and excitations (cf. Table II) that can be either realized by a separate Tx antenna or by using an active array that offers the flexibility to use parts of the $\mathrm{Rx}$ antenna for transmit. The scenarios show a resolution $\left(B_{P}=4.1\right.$ $\mathrm{kHz}$ ) equivalent to the dish antenna system and are compared to the "classical" system, where the Tx and Rx aperture have the same size what results in a slightly improved resolution of $1.65 \mathrm{~m}$. The resulting ambiguity suppression in Fig. 6 is worst for the uniform excitation and is already improved by more than $10 \mathrm{~dB}$ using a $6.2 \mathrm{~m}$ antenna with a simple triangular tapering and finally results in the quasi-optimum - as it approxi-

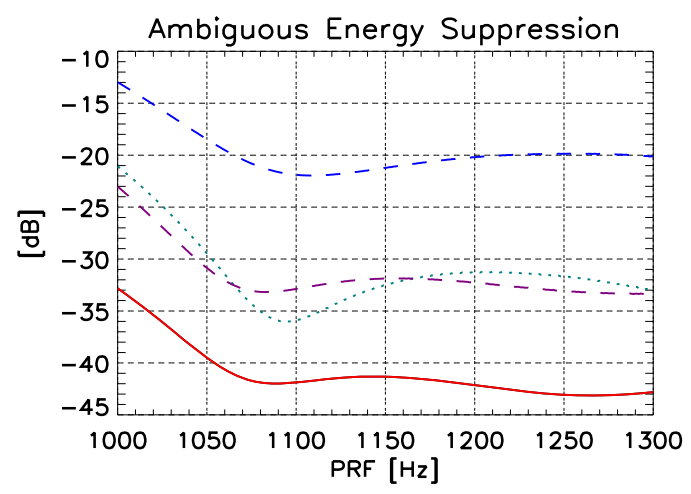

Fig. 6. Ambiguous Energy Suppression with pattern tapering: The "rectangular" pattern (solid red), a separate Tx dish (dashed violet), the triangular (dotted green) and the uniform excitation (dashed blue). The PRF from 1 to 1.3 $\mathrm{kHz}$ covers the relevant interval from 1100 to $1250 \mathrm{~Hz}$ w.r.t. the timing.

\begin{tabular}{|c|c|c|c|}
\hline Length & Excitation & Pattern & Resolution \\
\hline $5 \mathrm{~m}($ dish $)$ & uniform & Bessel & $1.75 \mathrm{~m}$ \\
\hline $14 \mathrm{~m}$ & $\sin (x) / x$ & Rectangular & $1.75 \mathrm{~m}$ \\
\hline $6.2 \mathrm{~m}$ & triangular & $\left(\sin \left(f_{x}\right) / f_{x}\right)^{2}$ & $1.75 \mathrm{~m}$ \\
\hline $2.8 \mathrm{~m}$ & uniform & $\sin \left(f_{x}\right) / f_{x}$ & $1.65 \mathrm{~m}$ \\
\hline
\end{tabular}

mates a rectangular pattern $-\sin (x) / x$ excitation. The plot is completed by the curve of the separate dish antenna, that runs similarly to the $\left(\sin \left(f_{x}\right) / f_{x}\right)^{2}$ pattern but with less variation vs. $P R F$. Especially the unconventional $\sin (x) / x$-excitation demonstrates the potential of tapering to efficiently cancel the spurious spectral components while preserving the resolution. A full exploitation of the benefits of tapering requires a fine adjustment of antenna dimensions, $P R F$ and $B_{P}$, taking into account the trade-off between resolution and ambiguity suppression.

\subsection{Pre-Beamshaping on Receive}

In contrast to 3.1, where the Tx branch is adapted to optimize the system performance, the following section investigates an innovative approach to effect the optimization by adjusting the $\mathrm{Rx}$ branch. The technique is called Pre-Beamshaping on Receive and is based on an Rx antenna consisting of a large number of individual apertures. These apertures are followed by a network that allows for an individual and reconfigurable combination of the -already amplified- apertures' RF signals. Then A/D conversion is performed yielding a number of adapted “channels" with optimized properties (cf. Fig. 7).

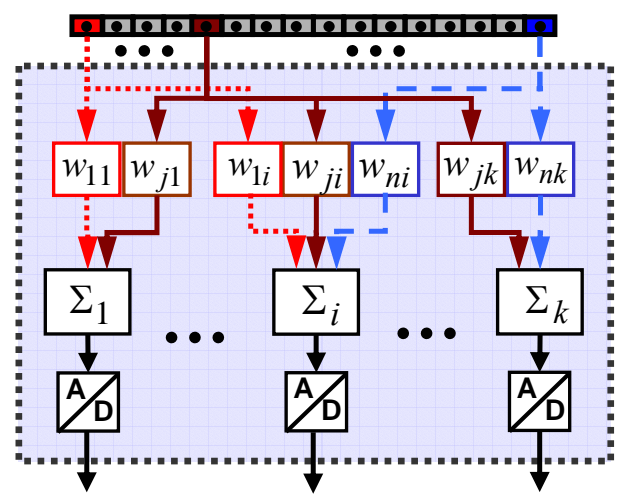

Fig. 7. "Pre-Beamshaping on Receive" network. Amplified RF signals of various apertures are adaptively weighted and combined before A/D conversion yields the optimized output channels.

In the following we consider again the "classical” system consisting of a Tx aperture of $2.8 \mathrm{~m}$ and 5 apertures on $\mathrm{Rx}$ of 2.8 $\mathrm{m}$ each. This system is compared to 3 different scenarios that are all based on the same Tx antenna and overall Rx antenna length, but the individual sub-apertures are realized by different Pre-Beamshaping on Receive implementations (cf. Table III). As indicated in Fig. 8, partly overlapping parts of the size $d_{r x, \text { eff }}$ of the Rx antenna are weighted with a triangular tapering yielding 5 sub-antennas with its phase centre in the middle of the respective sub-aperture. This means that the phase centres lie more closely, the larger the effective sub-aperture size $d_{r x \text {,eff }}$ is, as the overall antenna size remains constant. Hence, an appropriate choice of $d_{r x, \text { eff }}$ allows for setting the optimum PRF by adjusting the phase centre spacing, what enables an effective mitigation of the scaling of output noise power around the 


\begin{tabular}{|c|c|c|c|c|}
\hline $\boldsymbol{d}_{r \times, \text { eff }}$ & Tapering (Rx) & Phase Centre Spacing & $\boldsymbol{P R F}_{\text {uni }}$ & Resolution \\
\hline $2.8 \mathrm{~m}$ & uniform & $2.8 \mathrm{~m}$ & $1085 \mathrm{~Hz}$ & $1.65 \mathrm{~m}$ \\
\hline $4.4 \mathrm{~m}$ & triangular & $2.4 \mathrm{~m}$ & $1267 \mathrm{~Hz}$ & $1.66 \mathrm{~m}$ \\
\hline $5.6 \mathrm{~m}$ & triangular & $2.1 \mathrm{~m}$ & $1450 \mathrm{~Hz}$ & $1.72 \mathrm{~m}$ \\
\hline $6.4 \mathrm{~m}$ & triangular & $1.9 \mathrm{~m}$ & $1600 \mathrm{~Hz}$ & $1.79 \mathrm{~m}$ \\
\hline
\end{tabular}

TABLE III. Effective sub-aperture size $d_{r x, \text { eff }}$ with tapering, respective phase centre spacing with corresponding $P R F_{u n i}$ and resolution for $B_{p}=4.1 \mathrm{kHz}$.

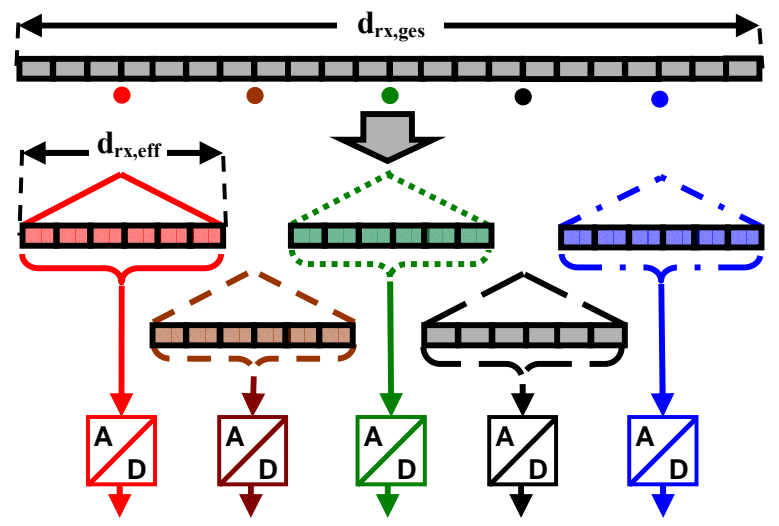

Fig. 8. Pre-Beamshaping on Receive: Mutually overlapping parts of the Rx antenna with triangular weighting form 5 virtual Rx apertures of size $d_{r x, e f f}$. The sub-apertures phase centers are indicated by the dots.

desired $P R F$. Fig. 9 shows the scaling of the output noise power normalized to the respective optimum value for the scenarios listed in Table III. One can observe that the scaling stays below $0.4 \mathrm{~dB}$ over a range of $700 \mathrm{~Hz}$ by flexibly reconfiguring the beamshaping network four times in dependency of the $P R F$ to adaptively adjust the overall sampling of the scenario. The maximum scaling can be further reduced by adapting the prebeamshaping more often. An effect to be considered in this context is a possible influence of the beamshaping network on the system noise caused by using some apertures' signals in more than one virtual channel due to the overlap of the subapertures. This topic is currently under investigation.

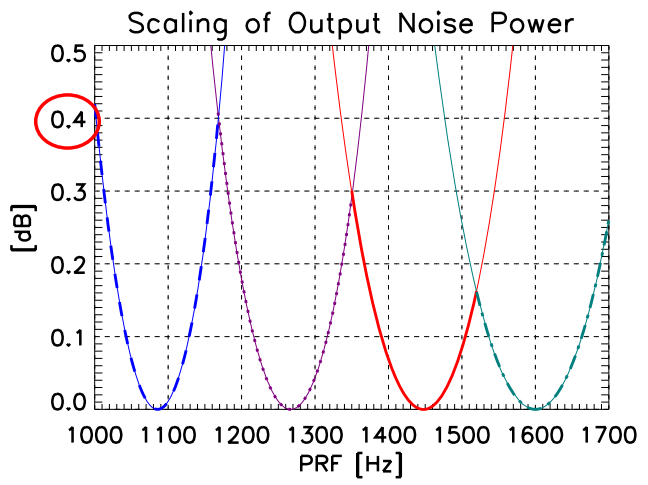

Fig. 9. Output Noise Scaling vs. $P R F$ for different beamshaping scenarios adapted to a $P R F$ of $1085 \mathrm{~Hz}$ (dashed blue), $1267 \mathrm{~Hz}$ (dotted violet), $1450 \mathrm{~Hz}$ (solid red) and $1600 \mathrm{~Hz}$ (dotted-dashed green), each normalised to its respective optimum. The effect of over-sampling is not considered in this plot.

A further effect of the adaptation of the phase centre to the $P R F$ and the consequentially reduced non-uniformity of the sampling is an improved ambiguity suppression. In addition, the larger effective sub-aperture size $d_{r x, \text { eff }}$ allows for pattern tapering on $\mathrm{Rx}$ and thus for reducing clearly the ambiguous energy in the respective $P R F$ range (cf. Fig. 10). These benefits come at the cost of a slightly increased resolution (cf. Table III).

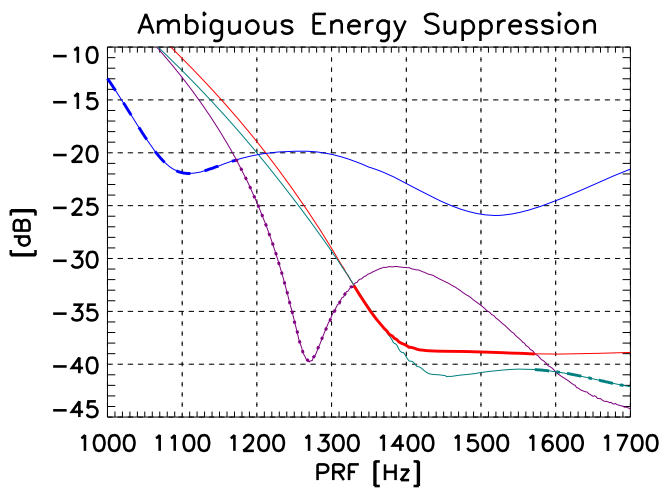

Fig. 10. Ambiguous Energy Suppression vs. PRF for different beamshaping scenarios adapted to $P R F$ regions around $1085 \mathrm{~Hz}$ (dashed blue), $1267 \mathrm{~Hz}$ (dotted violet), $1450 \mathrm{~Hz}$ (solid red) and $1600 \mathrm{~Hz}$ (dotted-dashed green).

\section{Discussion}

A comparison of azimuth processing methods for multiaperture signals showed that only the reconstruction algorithm and the null-steering approach provide high resolution in combination with efficient ambiguity suppression. For these approaches, a modified system with improved ambiguity suppression was presented. Further, optimization strategies were investigated showing the potential of pattern tapering of the Tx antenna to reduce ambiguities while preserving the resolution. Regarding the optimization on receive, first results for prebeamshaping on receive networks were presented, demonstrating the potential to adapt the system's virtual sampling to the $P R F$ and thus mitigating the effect of output noise scaling and, in combination with pattern tapering on $\mathrm{Rx}$, leading to an improved ambiguity suppression.

\section{References}

[1] G.D. Callaghan and I.D. Longstaff, Wide Swath Spaceborne SAR Using a Quad Element Array, IEE Radar Sonar and Navigation 146(3), pp 159-165, 1999

[2] M. Suess, B. Grafmüller, R. Zahn, A Novel High Resolution, Wide Swath SAR System, IGARSS 2001

[3] M. Younis, C. Fischer, W. Wiesbeck, Digital Beamforming in SAR systems, IEEE Trans. Geosci. Remote Sensing 41 (7), 2003

[4] L. Brule, H. Baeggli, Radarsat-2 Program Update, IGARSS 2002.

[5] J. Mittermayer, H. Runge, Conceptual Studies for Exploiting the TerraSAR-X Dual Receiving Antenna, IGARSS 2003.

[6] N.A. Goodman, S.C. Lin, D. Rajakrishna, J.M. Stiles, Processing of MultipleReceiver Spaceborne Arrays for Wide Area SAR, IEEE Trans. Geoscience and Remote Sensing 40 (4), pp 841-852, 2002

[7] G. Krieger and A. Moreira, Potentials of Digital Beamforming in Bi- and Multistatic SAR, IGARSS 2003

[8] J.P. Aguttes, The SAR Train Concept: Required Antenna Area Distributed over $N$ Smaller Satellites, Increase of Performance ... , IGARSS 2003

[9] G. Krieger, N. Gebert, A. Moreira, Unambiguous SAR Signal Reconstruction from Non-Uniform Displaced Phase Centre Sampling, IEEE Geoscience and Remote Sensing Letters, Vol. 1, No. 4, October 2004

[10] G. Krieger, N. Gebert, A. Moreira, Unambiguous SAR Signal Reconstruction from Non-Uniform Displaced Phase Centre Sampling, IGARSS 2004

[11] N. Gebert, G. Krieger, A. Moreira, SAR Signal Reconstruction from Non-Uniform Displaced Phase Centre Sampling in the Presence of Perturbations, IGARSS ' 05

[12] N. Gebert, G. Krieger, A. Moreira, High Resolution Wide Swath SAR Imaging - System Performance and Influence of Perturbations, IRS' 05

[13] H. Li, H. Wang, T. Su, Z. Bao, Generation of Wide-Swath and High-Resolution SAR Images From Multichannel Small Spaceborne SAR System, IEEE Geoscience and Remote Sensing Letters, Vol. 2, No. 1, Jan. 05

[14] N. Gebert, G. Krieger, A. Moreira, High Resolution Wide Swath SAR Imaging with Digital Beamforming - Performance Analysis, Optimization, System Design, EUSAR 2006 